\title{
Fructooligosaccharide/Lactobacillus paracasei/Lactobacillus \\ rhamnosus/Lactobacillus acidophilus/Bifidobacterium lactis Probiotic Supplement
}

National Cancer Institute

\section{Source}

National Cancer Institute. Fructooligosaccharide/Lactobacillus paracaseilLactobacillus

rhamnosus/Lactobacillus acidophilus/Bifidobacterium lactis Probiotic Supplement. NCI

Thesaurus. Code C125658.

A synbiotic nutritional supplement containing fructooligosaccharides (FOS), which are linear chains of fructose units that are linked by beta (2-1) bonds, and probiotic cultures of Lactobacillus paracasei (L. paracasei), L. rhamnosus, L. acidophilus and Bifidobacterium lactis (B. lactis), with gastrointestinal (GI) protective and immunomodulating activities. Upon oral administration, the bacteria in this probiotic supplement help maintain adequate colonization of the GI tract and modulate the composition of the normal microflora. Upon colonization of the GI tract, the probiotic bacteria form a protective barrier that helps maintain the integ rity of the epithelial barrier. This will interfere with the attachment of pathogenic bacteria and other harmful substances, prevent inflammation and improve Gl function. Additionally, the reconstituted microflora may metabolize FOS, which may promote calcium mobilization into the bloodstream by maintaining a neutral $\mathrm{pH}$ in the lower gut. 